\title{
Functional helical silica nanofibers with coaxial mixed mesostructures for the fabrication of PtCo nanowires that display unique geometry-dependent magnetism
}

\author{
Nien-Chu Lai ${ }^{1}$, Sz-Chian Liou ${ }^{2}$, Wei-Chia Huang ${ }^{1}$, Yu-Tien Shen ${ }^{3}$, Cheng-Chung $\mathrm{Chi}^{3}$, Ming-Wen $\mathrm{Chu}^{2}$ and \\ Chia-Min Yang ${ }^{1,4}$
}

Helical mesoporous silicas are notable from a self-assembly and applications points of view. We report a novel confinement-free synthesis of chloropropyl-functionalized helical mesoporous silica nanofibers (CP-HMSNFs) possessing straight channels at the fiber center surrounded by concentric short-pitch helical channels. The chloropropyl groups are found mainly distributed at the central cylindrical portion of the fibers, allowing the selective inclusion of guest species to fabricate novel nanocomposite fibers. Moreover, the chloropropyl-functionalized nanofibers were applied as a hard template to fabricate helical platinum-cobalt (PtCo) alloy nanowires with small and narrowly distributed radii of gyration. The helical metal nanowires exhibited distinct ferromagnetic properties as compared with their straight counterpart.

NPG Asia Materials (2015) 7, e181; doi:10.1038/am.2015.39; published online 22 May 2015

\section{INTRODUCTION}

Helical mesoporous silicas ${ }^{1-11}$ are analogous to the self-assembled helical biomaterials in nature and have attracted a substantial amount of attention. These helical silicas are also promising hard templates for the preparation of helical nanowires composed of metals ${ }^{12-15}$ or other compositions that may possess unique optical, ${ }^{16,17}$ electromagnetic ${ }^{9,18}$ or other properties. Efforts to understand the formation mechanism of these helical nanostructures provide additional insights into the interplay of the thermodynamics and kinetics of the assembly of structure-directing surfactants and inorganic silicate species ${ }^{1,2,19,20}$ and may also lead to the design and synthesis of additional novel selfassembled nanomaterials with controllable mesostructures and morphologies, allowing for innovative applications.9,11,21

The first synthesis of helical mesoporous silica materials displaying a MCM-41-like two-dimensional (2D)-hexagonal structure was performed in a static two-phase acidic system. ${ }^{7}$ Helical 2D-hexagonal mesoporous silica nanofibers (HMSNFs) or nanorods were then synthesized in single-phase dilute solutions using chiral ${ }^{5}$ or achiral $^{2,20,22}$ surfactants. The helical formation is mainly driven by a reduction in the free energy of the surface, from straight micellar rods until the free energy is balanced by an increased bending energy. ${ }^{2,20}$ Alternatively, HMSNFs can be prepared by evaporation-induced selfassembly (EISA) in porous anodic alumina membranes. ${ }^{23,24}$ The confined EISA may result in helical and other thermodynamically more stable mesophases, the former generally being kinetically favored. Notably, materials with mixed mesostructures can also be prepared by the confined EISA, ${ }^{25,26}$ and HMSNFs containing straight channels (that is, columnar mesostructure $)^{23,27}$ at the center surrounded by layers of concentric helical channels can also be prepared using this method. As novel hard templates, the HMSNFs may provide unprecedented opportunities in fabricating complex functional nanostructures, contingent on whether methods for the selective inclusion of guest specie s into differently oriented channels could be developed. However, the range of parameters for the confined EISA of these HMSNFs is narrow, and the preparation method cannot guarantee the uniformity and continuity of both mesostructures throughout the fiber. ${ }^{28}$ To the best of our knowledge, this type of HMSNFs have never been prepared by other methods.

We report on the confinement-free synthesis of chloropropylfunctionalized HMSNFs (CP-HMSNFs) with coaxial helical/columnar mesostructures in a single-phase solution. We found that the utilization of a mixture of cationic and nonionic surfactants and the co-condensation with a functional silane-bearing short hydrophobic functional groups are crucial for the spontaneous formation of these HMSNFs. We further discovered that the $\mathrm{CP}$ groups are mainly distributed at the central portion of the fibers, developing the potential for selective inclusion. We demonstrated this process of selective inclusion by preparing nanocomposites containing iron oxide and platinum (Pt). Moreover, we fabricated helical platinum-cobalt (PtCo) alloy nanowires in CP-HMSNFs and observed the geometrydependent ferromagnetism of these metal nanowires with small diameters and narrowly distributed radii of gyration.

${ }^{1}$ Department of Chemistry, National Tsing Hua University, Hsinchu, Taiwan; ${ }^{2}$ Center for Condensed Matter Sciences, National Taiwan University, Taipei, Taiwan; ${ }^{3}$ Department of Physics, National Tsing Hua University, Hsinchu, Taiwan and ${ }^{4}$ Frontier Research Center on Fundamental and Applied Sciences of Matters, National Tsing Hua University, Hsinchu, Taiwan

Correspondence: Professor C-M Yang, Department of Chemistry, National Tsing Hua University, 101, Sec. 2, Kuang-Fu Road, Hsinchu 30013, Taiwan.

E-mail: cmyang@mx.nthu.edu.tw

Received 2 November 2014; revised 3 February 2015; accepted 16 March 2015 


\section{EXPERIMENTAL PROCEDURE}

\section{Synthesis of CP-HMSNFs}

The CP-functionalized samples were synthesized by injecting a mixture of tetraethoxysilane (TEOS, Acros, Geel, Belgium) and chloropropyltriethoxysilane (CPTES, Aldrich, St Louis, MO, USA) into a solution containing cetyltrimethylammonium bromide (CTAB, Acros), tetraethylene glycol dodecyl ether $\left(\mathrm{C}_{12} \mathrm{E}_{4}\right.$, Aldrich) and sodium hydroxide $(\mathrm{NaOH})$. The molar composition of the synthesis mixture was $\left(1-f_{C}\right)$ TEOS: $f_{C}$ CPTES: 0.09375 CTAB: 0.03125 $\mathrm{C}_{12} \mathrm{E}_{4}: 0.32 \mathrm{NaOH}: 1230 \mathrm{H}_{2} \mathrm{O}$, where $f_{C}$ denotes the fraction of CPTES in the silane mixture and was varied between $0 \%$ and $15 \%$. The injection rate of silane mixture was $3.0-15.0 \mathrm{ml} \mathrm{h}^{-1}$. The mixture was stirred at $35^{\circ} \mathrm{C}$ for $2 \mathrm{~h}$ and was then aged at $90^{\circ} \mathrm{C}$ for $24 \mathrm{~h}$. The product was filtered, washed with water and acetone and finally dried in air. In several syntheses, CPTES was replaced by iodopropyltrimethoxysilane (Fluka, Seelze, Germany) and trifluoropropyltrimethoxysilane (Alfa Aesar, Lancashire, UK). The surfactants in the assynthesized samples were removed by calcination at $540^{\circ} \mathrm{C}$ or solvent extraction in an acidified ethanol solution.

In addition, a MCM-41 sample was synthesized as a reference template for the fabrication of PtCo nanowires. The synthesis procedure was the same as that for the functionalized samples except for the molar composition of 1 TEOS: 0.125 CTAB: $0.32 \mathrm{NaOH}: 1230 \mathrm{H}_{2} \mathrm{O}$. The surfactants were removed by ethanol extraction.

\section{Incorporation of $\mathrm{Pt}$ in the calcined and extracted CP-HMSNFs}

The calcined or extracted sample of CP-HMSNFs $(0.40 \mathrm{~g})$ was mixed with chloroplatinic acid hexahydrate $\left(\mathrm{H}_{2} \mathrm{PtCl}_{6} \cdot 6 \mathrm{H}_{2} \mathrm{O}\right.$, Strem, Boston, MA, USA, $0.68 \mathrm{~g}$ ) in a glass vial under ambient conditions. The mixture was maintained at $80^{\circ} \mathrm{C}$ for $12 \mathrm{~h}$ to allow the molten salt to infiltrate into the mesopores of the sample. The mixture was heated at $200^{\circ} \mathrm{C}$ for $2 \mathrm{~h}$ in a stream of hydrogen to reduce the metal.

\section{Preparation of the nanocomposite of $\mathrm{Fe}_{2} \mathrm{O}_{3}$ and $\mathrm{Pt}$}

The extracted sample of CP-HMSNFs $(0.18 \mathrm{~g})$ was first mixed with iron (III) nitrate nonahydrate $\left(\mathrm{Fe}\left(\mathrm{NO}_{3}\right)_{3} \cdot 9 \mathrm{H}_{2} \mathrm{O}\right.$, Showa, Saitama, Japan, $\left.0.22 \mathrm{~g}\right)$ in a glass vial under ambient conditions. The mixture was maintained at $70^{\circ} \mathrm{C}$ for $12 \mathrm{~h}$ and was then heated to $250^{\circ} \mathrm{C}$ for $3 \mathrm{~h}$. The incorporation was repeated again with $0.20 \mathrm{~g}$ of $\mathrm{Fe}\left(\mathrm{NO}_{3}\right)_{3} \cdot 9 \mathrm{H}_{2} \mathrm{O}$. The sample was heated at $540{ }^{\circ} \mathrm{C}$ for $6 \mathrm{~h}$ to form $\mathrm{Fe}_{2} \mathrm{O}_{3}$ in the mesopores and simultaneously decompose the CP groups. Subsequently, Pt was incorporated into the $\mathrm{Fe}_{2} \mathrm{O}_{3}$-loaded sample $(0.05 \mathrm{~g})$ by the procedure described above for $\mathrm{H}_{2} \mathrm{PtCl}_{6} \cdot 6 \mathrm{H}_{2} \mathrm{O}(0.04 \mathrm{~g})$.

Incorporation of PtCo in the extracted CP-HMSNFs and MCM-41 The extracted sample of CP-HMSNFs or MCM-41 (0.050 g) was mixed with a solution containing $\mathrm{H}_{2} \mathrm{PtCl}_{6} \cdot 6 \mathrm{H}_{2} \mathrm{O}(0.092 \mathrm{~g}$ ), cobalt (II) nitrate hexahydrate (Showa, $0.050 \mathrm{~g}$ ) and water $(0.2 \mathrm{ml})$. The mixture was dried at $60^{\circ} \mathrm{C}$ under reduced pressure and was then maintained at $80^{\circ} \mathrm{C}$ for $12 \mathrm{~h}$. The metals in the resulting solid were reduced using hydrogen at $350^{\circ} \mathrm{C}$ for $3 \mathrm{~h}$.

\section{Characterization}

X-ray diffraction (XRD) patterns were recorded on a Mac Science 18MPX diffractometer using $\mathrm{Cu} K \alpha$ radiation. Solid-state ${ }^{29} \mathrm{Si}$ MAS NMR spectra were measured on a Bruker AVANCE III spectrometer using a 4-mm MAS probe. Nitrogen physisorption isotherms were measured at $77 \mathrm{~K}$ using a Quantachrome Autosorb-1MP instrument (Boynton Beach, FL, USA). The isotherms were analyzed by nonlocal density functional theory (by applying a $\mathrm{N}_{2}(77 \mathrm{~K})$ kernel based on cylindrical pore geometry) to evaluate the pore size and surface area of the samples. The total pore volume was evaluated at a relative pressure $\left(\mathrm{P} / \mathrm{P}_{0}\right)$ of 0.9 . Scanning electron microscope images were obtained with a field emission JEOL JSM-7000 F microscope operating at $10 \mathrm{kV}$. The samples were coated with Pt before the measurements. Transmission electron microscope (TEM) images were taken using a JEOL JEM-2010 microscope operated at $200 \mathrm{kV}$. Scanning transmission electron microscopy (STEM) images and tomography experiments were conducted on a field-emission microscope operated at $200 \mathrm{keV}$ (FEI F20 G2) with an electron-probe size of $\sim 2 \AA$. The tilt series for each tomography study consists of 141 STEM-HAADF images, which were acquired from the tilting of the angles from $-70^{\circ}$ to $+70^{\circ}$. Typically, images are recorded every $1^{\circ}$. The three-dimensional (3D) reconstructions of the 2D STEM-HAADF data sets were performed using a weighted back projection algorithm integrated in the FEI Inspect3D (Hillsboro, OR, USA) software package, and further 3D visualizations were conducted using AMIRA 4.0 (Mercury Computer Systems). Energy dispersive X-ray spectroscopy measurements were conducted using an Oxford EDX system. The magnetization was measured in a SQUID VSM magnetometer (Quantum Design, San Diego, CA, USA) from 5 to $300 \mathrm{~K}$. The isothermal magnetization was measured with a solenoid in the hysteresis mode swept to $\pm 5 \mathrm{kOe}$. Circular dichroism measurements were conducted on an Aviv Model 410 circular dichroism spectrometer.

\section{RESULTS AND DISCUSSION}

The CP-HMSNFs were synthesized by injecting the mixture of TEOS and CPTES into a dilute and alkaline solution of CTAB and $\mathrm{C}_{12} \mathrm{E}_{4}$ with a molar ratio of $\mathrm{CTAB}: \mathrm{C}_{12} \mathrm{E}_{4}=7: 3$. The synthesis systems using mixed cationic and nonionic surfactants are versatile in forming highly ordered mesoporous silicas with mesostructures in between 2Dhexagonal and lamellar phases and with unique morphologies. ${ }^{3,4,29,30}$ The XRD patterns displayed that although the injection of TEOS alone with a rate of $3.0 \mathrm{ml} \mathrm{h}^{-1}$ led to a 2Drectangular MMT-1 material ${ }^{3}$ (with unit cell parameters $a$ and $b$ of $10.9 \mathrm{~nm}$ and $4.5 \mathrm{~nm}$, respectively), the partial replacement of TEOS by CPTES caused a transformation to a 2D-hexagonal structure (Figure 1a). The structural transformation was almost complete when the fraction of CPTES in the silane mixture $\left(f_{C}\right)$ reached $5 \%$, and the XRD patterns of the samples synthesized with $f_{C}=10$ and $15 \%$ could be perfectly indexed with a $p 6 \mathrm{~mm}$ lattice. The sample with $f_{C}=10 \%$ showed the best structural order, as indicated by the most intense and sharpest reflections. The solid-state ${ }^{29}$ Si MAS NMR analysis suggested that the ratios of intensity, $\mathrm{T}^{\mathrm{n}} / \mathrm{T}^{\mathrm{n}}+\mathrm{Q}^{\mathrm{m}}\left(\mathrm{T}^{\mathrm{n}}\right.$ groups: $\left(\mathrm{Si}(\mathrm{OSi})_{\mathrm{n}}(\mathrm{OH})_{3}\right.$ $\left.{ }_{n} \mathrm{C}\right)$; $\mathrm{Q}^{\mathrm{m}}$ groups: $\left.\left(\mathrm{Si}(\mathrm{OSi})_{\mathrm{m}}(\mathrm{OH})_{4-\mathrm{m}}\right)\right)$, are nearly identical to $f_{C}$ in the silane mixtures (Supplementary Figure $S 1$ in the Supplementary Information). After the surfactants removal by solvent extraction or calcination, the surfactant-free samples exhibited type IV nitrogen physisorption isotherms with sharp steps over the relative pressure range of 0.25-0.4 (Supplementary Figure S2). The textural properties were similar for the samples with $f_{C}=0-15 \%$, and the derived values of the mesopore diameters, surface areas and pore volumes are $2.5-2.7 \mathrm{~nm}, 1195-1280 \mathrm{~m}^{2} \mathrm{~g}^{-1}$ and $0.96-1.01 \mathrm{~cm}^{3} \mathrm{~g}^{-1}$, respectively.

Notably, the transformation was accompanied by changes in particle morphology. Scanning electron microscope images showed that although the pure silica sample $\left(f_{C}=0 \%\right)$ mainly contained spherical particles with sizes of 350-600 nm (Supplementary Figure S3), thin fibers started to appear in the sample with $f_{C}=5 \%$. The fraction of nanofibers increased with increasing $f_{C}$. More than $90 \%$ of the particles were CP-HMSNFs (with diameters of $60-110 \mathrm{~nm}$ ) in the sample with $f_{C}=10 \%$ (Figure $1 \mathrm{~b}$ ). Additionally, the samples with $f_{C}=15 \%$ consisted exclusively of CP-HMSNFs (Supplementary Figure S3). The interior structure of the CP-HMSNFs in the sample with $f_{C}=10 \%$ was further revealed in TEM images. As shown in Figure 1c, the projection of the hexagonally packed channels is observed only at both edges of the nanofiber, suggesting that the channels were wound around the center axis of the nanofiber. Similar projection images have been observed for MMT-1 materials with short helical pitch. ${ }^{3,4}$ The injection rate of the silane mixture also affected the morphology of the resulting sample without modifying the $2 \mathrm{D}$-hexagonal mesostructure. When $f_{C}=10 \%$, an increase of the injection rate (up to 15.0 $\mathrm{ml} \mathrm{h}^{-1}$ ) resulted in samples with smaller fractions of nanofibers that were thinner and shorter than those synthesized with an injection rate of $3.0 \mathrm{ml} \mathrm{h}^{-1}$ (Supplementary Figure S4). 

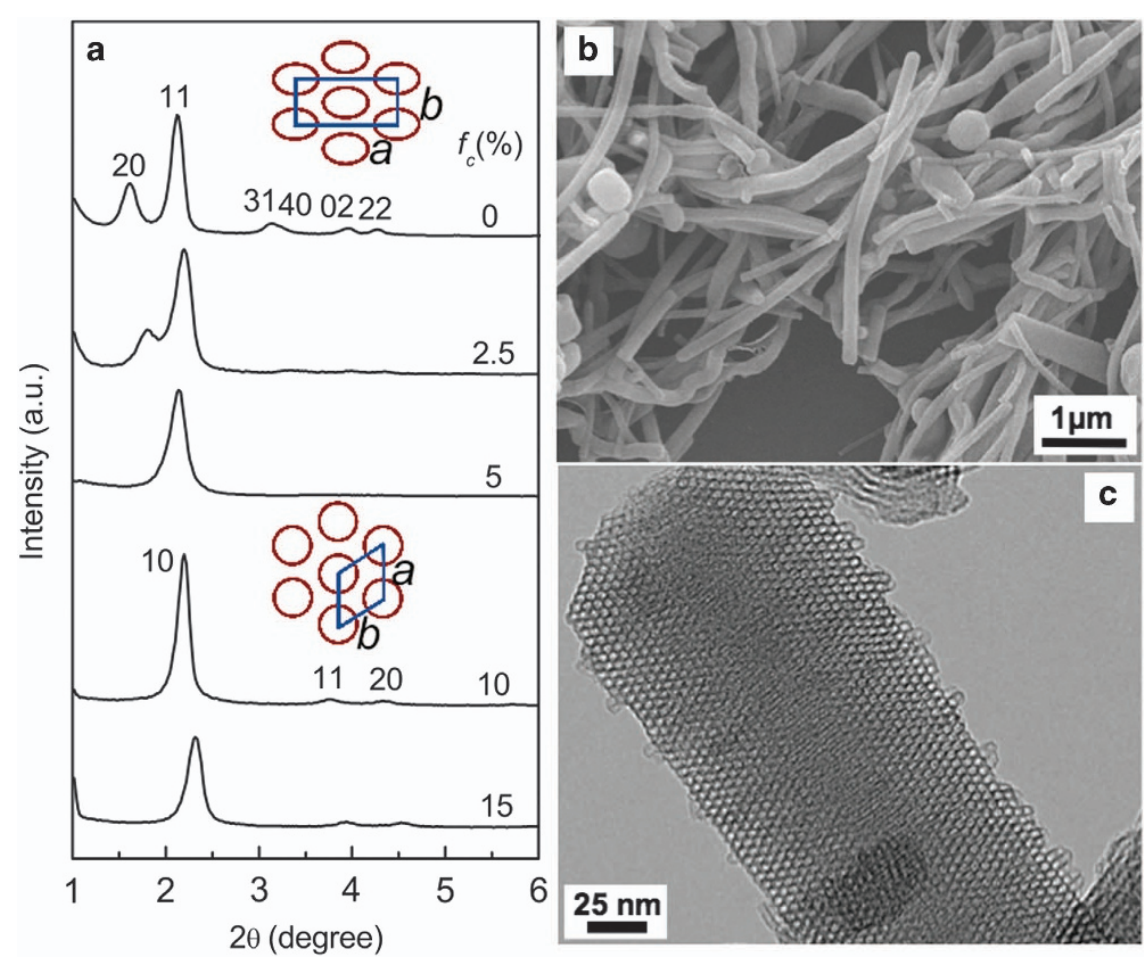

Figure 1 (a) XRD patterns of the samples synthesized with a varied fraction of CPTES in the silane mixture ( $f_{C}$ ). (b and $\mathbf{c}$ ) Typical SEM and TEM images of the sample with $f_{C}=10 \%$. a.u., arbitrary unit; CPTES, chloropropyltriethoxysilane; SEM, scanning electron microscope; TEM, transmission electron microscope; XRD, X-ray diffraction.

To further reveal the orientation of the channels in the functional mesoporous nanofibers, we incorporated large amounts of $\mathrm{Pt}$ (40 wt $\%)$ into the surfactant-free CP-HMSNFs for TEM and highangle annular dark-field (HAADF) imaging with STEM using the characteristic atomic number-dependent contrast (Z-contrast) for HAADF. The incorporation was achieved by a method we recently developed that involves impregnation with molten hydrated platinic acid followed by hydrogen reduction. ${ }^{31}$ We first investigated the Ptinfiltrated calcined CP-HMSNFs. The bright-field TEM images (Supplementary Figure S5) show that almost all of the metal was incorporated inside the channels to form continuous nanowires, allowing us to visualize the channels and study their orientation in the nanofibers. Surprisingly, a series of images with different tilting angles showed the coexistence of helical and straight Pt nanowires in the fibers, with the former coiling around the latter at the fiber center. Similar types of mixed helical/columnar mesostructures have only been fabricated by confined EISA in anodic alumina membranes. ${ }^{23,27}$ In Figure 2a, seven consecutive STEM-HAADF images are stitched together to show that the mixed mesostructures are coaxial and extend throughout a fiber. We further employed HAADF-STEM tomography to unveil the $3 \mathrm{D}$ organization of $\mathrm{Pt}$ nanowires in the composite channel systems of the CP-HMSNFs. A tilt series of $\sim 140 \mathrm{HAADF}$ images was taken for a $3 \mathrm{D}$ reconstruction with the tilt range within $\sim \pm 70^{\circ}$. We demonstrated the analytical results of two Pt-filled fiber segments. Figure $2 \mathrm{~b}$ and Supplementary Figure S6 show the HAADF images from the tilt series of the first segment, in which the changes in $2 \mathrm{D}$ projections can be observed. Figure $2 \mathrm{c}$ shows snapshots of the $3 \mathrm{D}$ reconstructed images of the same segment rotated around the fiber axis by $0^{\circ}, 90^{\circ}, 180^{\circ}$ and $270^{\circ}$. With a resolution of $\sim 1 \mathrm{~nm}$ in all directions, ${ }^{32}$ the images showed that both the helical and straight $\mathrm{Pt}$ nanowires were 3 -nm wide, a value consistent with the mesopore diameter estimated by the nitrogen physisorption. The helical nanowires display short pitches of approximately $3-5 \mathrm{~nm}$, and the radii of gyration of the most inner and the most outer nanowires are $12 \mathrm{~nm}$ and $55 \mathrm{~nm}$, respectively. We further compared the cross-sectional images of the segment at the specified positions perpendicular to the fiber axis (Figure 2d). The streaks in Figure 2d mainly arise from the missing-wedge effect and do not noticeably affect the $3 \mathrm{D}$ reconstruction, as demonstrated by the consistency between Figure 2d and its 2D counterpart in Figure $2 \mathrm{~b}$. Seven straight Pt nanowires are noted at the center of the fiber, forming the smallest hexagonal columnar domain. The same small and well-defined central columnar domain was observed in all analyzed fibers, suggesting that its formation should be governed by certain thermodynamic and/or kinetic factors. We further analyzed another segment containing fewer Pt nanowires to study the handedness of the helical channels. The representative HAADF and reconstructed images are shown in Figure 2e, and the high-resolution video files of the segment rotating along and perpendicular to the fiber axis are available in the Supplementary Information (Supplementary Movie S1 and S2). All helical Pt nanowires in the segment are right-handed, regardless of the radius of gyration. Based on the analysis of tens of Pt-infiltrated fiber segments and the circular dichroism spectrum of the sample (Supplementary Figure S7), we conclude that all channels in each CP-HMSNF possess the same chirality and that the samples are racemic and contain approximately the same fraction of right-handed and left-handed helical fibers.

We then attempted to determine the distribution of CP (or chloropropylsilyl (CPS)) groups in the CP-HMSNFs to provide insights into the formation of HMSNFs with unique mixed mesostructures. We prepared another surfactant-free sample of CP-HMSNFs by solvent extraction for Pt infiltration. Because CP 

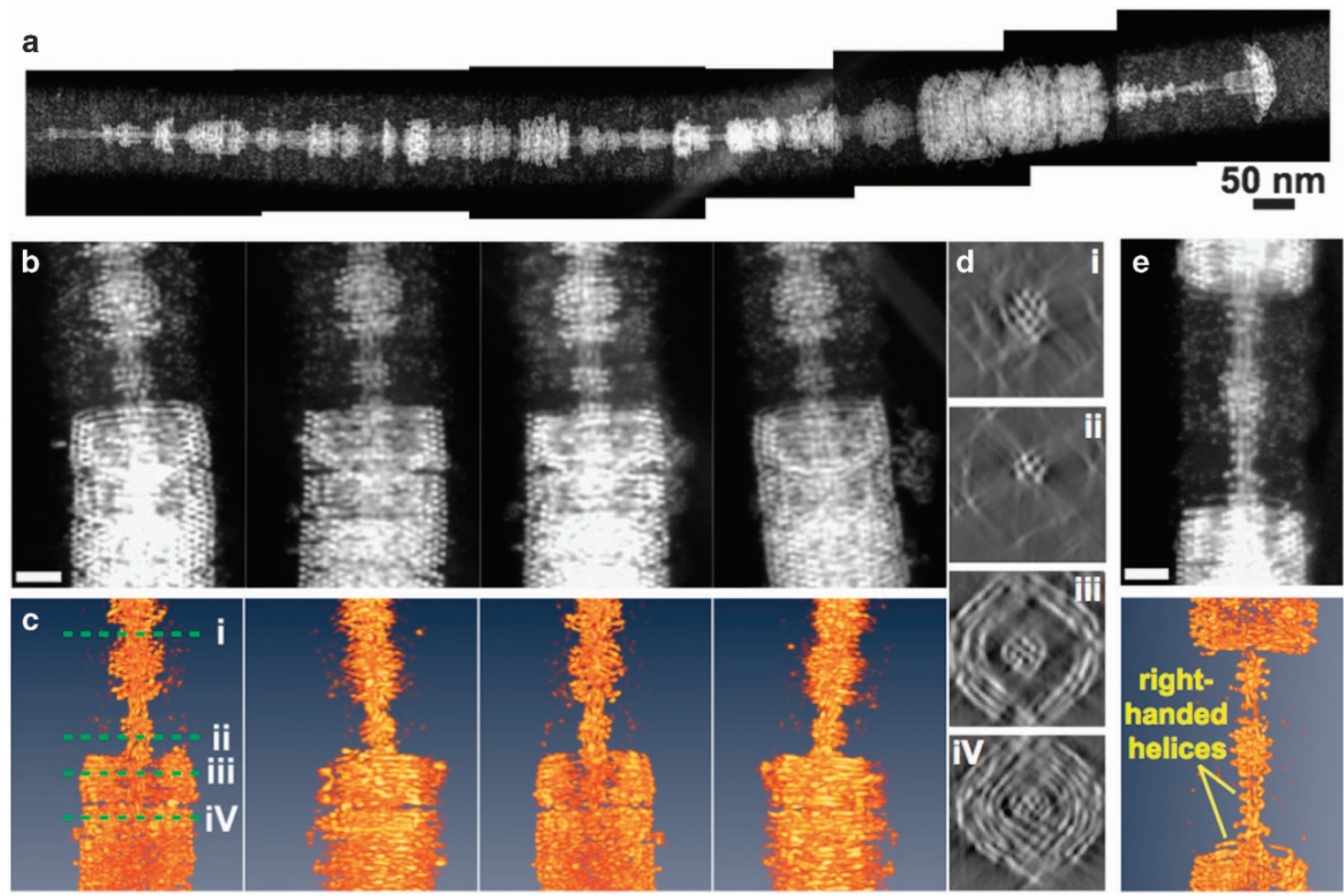

Figure 2 (a) Seven consecutive STEM-HAADF images of the Pt-infiltrated calcined CP-HMSNFs showing that the coaxial helical and straight channels extend throughout the fiber. (b) A series of STEM-HAADF images of a segment of Pt-filled fiber tilted around the fiber axis by (from left to right) $+40^{\circ},+10^{\circ},-10^{\circ}$ and $-40^{\circ}$. (c) $3 \mathrm{D}$ reconstructed images of the same segment rotated around the fiber axis by (from left to right) $0^{\circ}, 90^{\circ}, 180^{\circ}$ and $270^{\circ}$. (d) Cross-sectional images of the segment shown in the first frame of $\mathbf{c}$ at the indicated positions revealing that the helical Pt nanowires are coiled around straight Pt nanowires at the fiber center. The streaks in $\mathbf{d}$ mainly arise from the missing-wedge effect and do not noticeably affect the 3D reconstruction, as demonstrated by the consistency between $\mathbf{d}$ and its 2D counterpart in $\mathbf{b}$. (e) A STEM-HAADF image (top) with the corresponding reconstructed image (bottom) of another segment of Pt-filled fiber showing the same chirality of Pt nanowires in the helical channels. The scale bars in $\mathbf{b}$ and $\mathbf{e}$ indicate $30 \mathrm{~nm}$. Video files of the segment shown in e rotating along and perpendicular to the fiber axis are available in the online (HTML) version of this article. CP-HMSNF, chloropropylfunctionalized helical mesoporous silica nanofiber; HAADF, high-angle annular dark-field; Pt, platinum; STEM, scanning transmission electron microscopy; 2D, two-dimensional; 3D, three-dimensional.

groups are hydrophobic, the molten platinic acid infiltrates and is reduced only in channels in which the organic groups are absent or loosely distributed. Figures $3 \mathrm{a}$ and $\mathrm{b}$ show typical bright-field and HAADF images of the Pt-infiltrated $(\sim 40 \mathrm{wt} \%)$ extracted CP-HMSNFs. Surprisingly, we found that almost no Pt was filled in the central cylindrical part of every fiber that expands from the fiber axis to a distance of approximately $30-40 \mathrm{~nm}$, and helical Pt nanowires were observed at the periphery of the fibers (Figure 3c). These observations strongly suggest that most of the CPS groups were located at the central cylindrical part of the CP-HMSNFs. HMSNFs with spatially localized functionality have never been reported, and this type of HMSNFs may allow the fabrication of nanocomposite fibers that display selective inclusion. To demonstrate the feasibility of this process, we prepared a nanocomposite of iron oxide ( $\alpha$-phase $\mathrm{Fe}_{2} \mathrm{O}_{3}$, see Supplementary Figure S8 for the XRD patterns) and $\mathrm{Pt}$ by incorporating repeatedly the molten ferric nitrate (with a melting point of $47^{\circ} \mathrm{C}$ ) into the extracted CP-HMSNFs, transforming the salt to $\mathrm{Fe}_{2} \mathrm{O}_{3}$ whereas decomposing the $\mathrm{CP}$ groups by thermal treatment and finally loading $\mathrm{Pt}$ using the aforementioned procedure. Figure 4 shows a STEM-bright-field image of a segment of the nanocomposite fiber and the corresponding STEM-energy dispersive X-ray spectroscopy element maps. As expected, we found that the iron species were mainly located at the periphery of the fiber whereas straight and helical Pt nanowires were at the center of the fiber. With this demonstration, further design and fabrication of novel nanocomposite helical fibers can be envisioned.

As the first example of the confinement-free synthesis of HMSNFs with mixed mesostructures, our discovery illustrates the versatility of the surfactant-directed cooperative assembly to form novel inorganic nanomaterials. ${ }^{10,11}$ To understand the development of the unique mesostructures, morphology and localized functionality of CP-HMSNFs, we investigated the possible influence of the silane CPTES on the assembly process. The CP moiety of the CPS group is relatively short and hydrophobic, and the hydrolysis of CPTES is faster than that of TEOS because of the inductive effect of the CP group. ${ }^{33}$ Therefore, when the silane mixture (with $f_{C}=10-15 \%$ ) was injected into the binary surfactants solution, CPTES molecules were quickly hydrolyzed to produce the corresponding anionic silicate species. These silicate species would then be readily adsorbed onto the CTA cations in the micelles of the binary surfactants with the $\mathrm{CP}$ group intercalated into the hydrophobic core of the micelles. Therefore, the initially formed micellar rods would be mainly encased with the CPTES-derived CPS-containing species, as represented in Scheme 1. The hydrolytic condensation of these silicate species would then cause the encased micellar rods to bend, coil and form highly curved and short-pitch helices, a process driven by the significant reduction in surface free energy but accompanied with an increase in the bending 

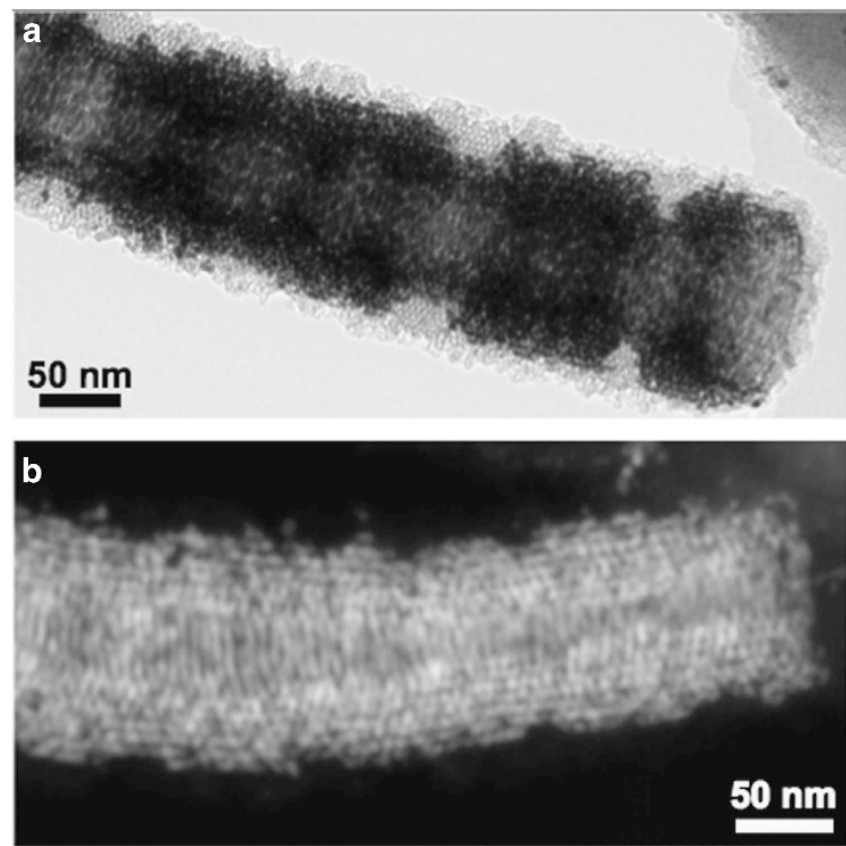

c

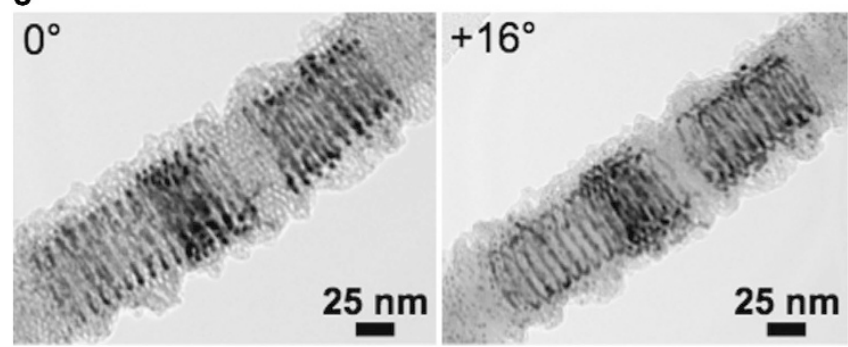

Figure 3 ( $\mathbf{a}$ and $\mathbf{b}$ ) BF-TEM and STEM-HAADF images of the Pt-infiltrated extracted CP-HMSNFs. (c) BF-TEM images with different tilting angles showing that helical Pt nanowires are at the periphery of the fiber. BF, bright field; CP-HMSNF, chloropropyl-functionalized helical mesoporous silica nanofiber; HAADF, high-angle annular dark-field; Pt, platinum; STEM, scanning transmission electron microscopy; TEM, transmission electron microscopy.

energy of micellar rods. ${ }^{2-4,20}$ Subsequently, the fusion of encased micelles at the ends of the helix led to the elongation of the helices, whereas the stacking of the encased micellar rods onto the helices resulted in a further growth in width. For the synthesis with $f_{C}=10$ $15 \%$, the elongation of the helices (longitudinal growth) should proceed much faster than transverse growth to result in thin nanofibers. Because a significant amount of the micelles mainly encased with CPS-containing species were formed faster owing to the faster hydrolysis of the CPTES and the additional hydrophobic interactions of the $\mathrm{CP}$ groups with the micellar core, these micelles might preferentially fuse to the ends of the innermost and highly curved helices to contribute to the longitudinal fiber growth. This phenomenon may explain why the CPS groups are mainly distributed at the center of the fibers. In addition, the densely distributed CPS-containing anionic species may also reduce the charge density of the head groups of the CTA cations, thereby destabilizing the highly curved and bent micellar rods at the fiber center and triggering the transformation into columnar hexagonal packing to release the micellar bending energy. ${ }^{24,28}$ The coaxial helical/columnar mesostructures then continued to grow simultaneously to produce the nanofibers observed in the final product. The proposed formation mechanism of functional HMSNFs may also occur for other trialkoxysilanes bearing short and hydrophobic groups. To examine this process, we applied the same synthesis conditions (with $f_{C}=10 \%$ ) to prepare materials using iodopropyltrimethoxysilane and trifluoropropyltrimethoxysilane. The resulting samples mainly contained HMSNFs with mixed mesostructures (Figure 5). However, the syntheses using silanes with short polar groups (for example, mercaptopropyl group) or longer hydrophobic groups (for example, octyl group, (Supplementary Figure S9)) mainly resulted in spherical particles.

With the hydrophobic groups localized at the center of fibers, CP-HMSNFs provide hydrophilic helical channels with a small and relatively narrow distribution of the radius of gyration for the template fabrication. In this study, we applied the extracted CP-HMSNFs to prepare bundles of 3-nm-wide helical PtCo alloy nanowires with radii of gyration of approximately $35-55 \mathrm{~nm}$ and studied the geometrydependent magnetic properties in comparison with the straight nanowires fabricated in MCM-41 with straight channels. Magnetic PtCo alloys are of great interest because of their large uniaxial magnetocrystalline anisotropy constants that cause ferromagnetic properties down to the nanometer scale; these properties are appropriate for ultra-high density storage and other innovative applications. ${ }^{9,11,34}$ The nanowires in the two samples had identical compositions of $60 \% \mathrm{Pt}$ and $40 \%$ Co as determined by energy dispersive X-ray spectroscopy, and they also had nearly identical lattice constants, degree of alloying and diameters as confirmed by XRD and TEM (Supplementary Figure S10 and S11) analyses. The nominal total metal loadings in both samples $\left(\mathrm{Pt}_{60} \mathrm{Co}_{40} @ \mathrm{CP}-\mathrm{HMSNF}\right.$ and $\left.\mathrm{Pt}_{60} \mathrm{Co}_{40} @ \mathrm{MCM}-41\right)$ were $33 \mathrm{wt} \%$. As shown in Figure 6, the magnetization-demagnetization curves indicate that both samples were ferromagnetic at $5-300 \mathrm{~K}$ and that complete saturation was almost achieved at a magnetic field of $5 \mathrm{~T}$. The derived magnetic properties are compiled in Table 1. The preparation of the two samples was repeated, and nearly the same magnetic performances (with a s.d.of $10^{-8}$ e.m.u.) were observed for three different batches of samples of the same type. At $5 \mathrm{~K}$, the saturation magnetization of $\mathrm{Pt}_{60} \mathrm{Co}_{40} @ \mathrm{CP}-\mathrm{HMSNFs}(0.469$ Bohr magnetons per formula unit $\left(\mu_{\mathrm{B}}\right.$ f.u. $\left.\left.{ }^{-1}\right)\right)$ is smaller than that of $\mathrm{Pt}_{60} \mathrm{Co}_{40} @ \mathrm{MCM}-41$ $\left(0.548 \mu_{\mathrm{B}}\right.$ f.u. $\left.^{-1}\right)$. This result is likely to be correlated to a smaller fraction of magnetic moments in $\mathrm{Pt}_{60} \mathrm{Co}_{40} @ \mathrm{CP}-\mathrm{HMSNF}$ that are capable of being aligned parallel to the applied magnetic field because of the helical nanowire geometry. ${ }^{35,36} \mathrm{Pt}_{60} \mathrm{Co}_{40} @ \mathrm{CP}-\mathrm{HMSNF}$ also exhibited smaller remanent magnetization, remanence ratio (saturation/remanent) and lower coercivity than $\mathrm{Pt}_{60} \mathrm{Co}_{40} @ \mathrm{MCM}-41$ did. Straight alloy nanowires are magnetically harder than helical nanowires, perhaps because of the stronger exchange interactions between the aligned magnetic domains in (and between) straight nanowires. ${ }^{37}$ However, the straight nanowires experienced a larger decrease ( $69 \%)$ in coercivity than helical nanowires did $(\sim 49 \%)$ when increasing the temperature from 5 to $300 \mathrm{~K}$. This phenomenon could be attributed to the higher Curie temperature for helical nanowires. ${ }^{37}$ The field-cooled magnetization curves (Supplementary Figure S12) showed that, although the Curie temperature of the two samples seemed to be well above the highest temperature $(300 \mathrm{~K})$ the magnetometer could reach, the helical nanowires seemed to display higher Curie temperatures than the straight counterpart, as suggested by the slower change in the susceptibility of the $\mathrm{Pt}_{60} \mathrm{Co}_{40} @ \mathrm{CP}-\mathrm{HMSNF}$ with respect to temperature at 100-300 K. To further confirm the observed geometrydependent magnetic properties, we prepared $\mathrm{Pt}_{60} \mathrm{Co}_{40}$ nanoparticles instead of nanowires in MCM-41 and CP-HMSNFs with the identical 


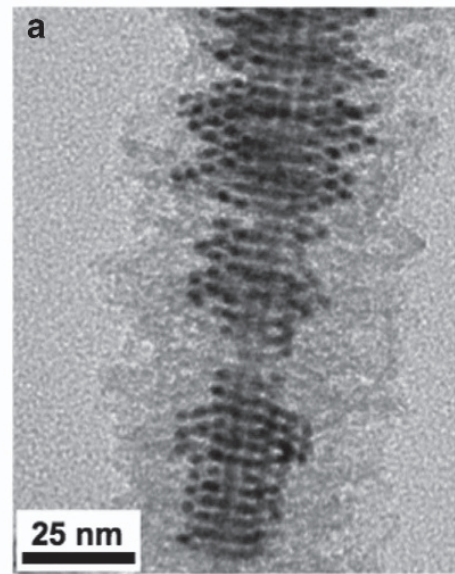

b
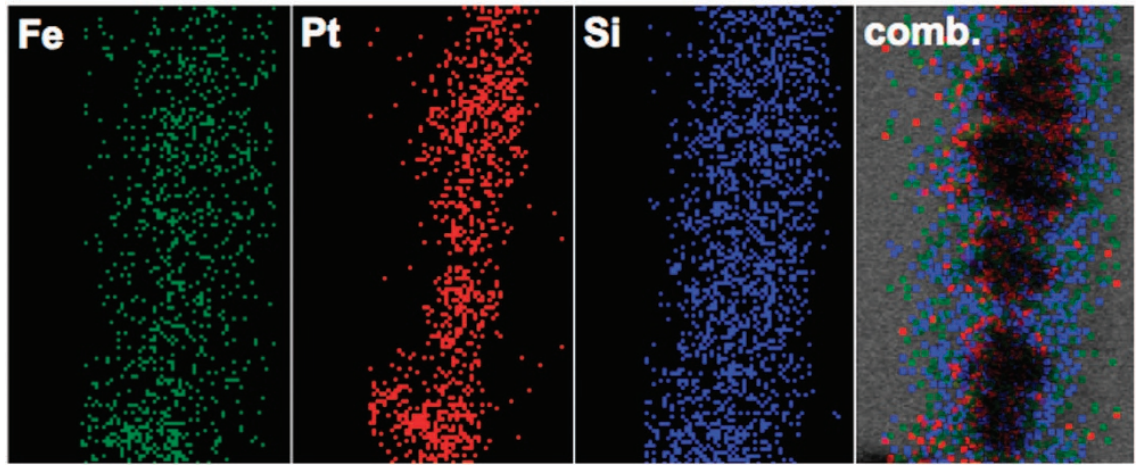

Figure 4 (a) BF-TEM image of a segment of the nanocomposite fiber containing $\alpha-\mathrm{Fe}_{2} \mathrm{O}_{3}$ and Pt. (b) STEM-EDX elemental mapping and the combination of the images with a. BF, bright field; EDX, energy dispersive X-ray spectroscopy; Pt, platinum; STEM, scanning transmission electron microscopy; TEM, transmission electron microscopy.

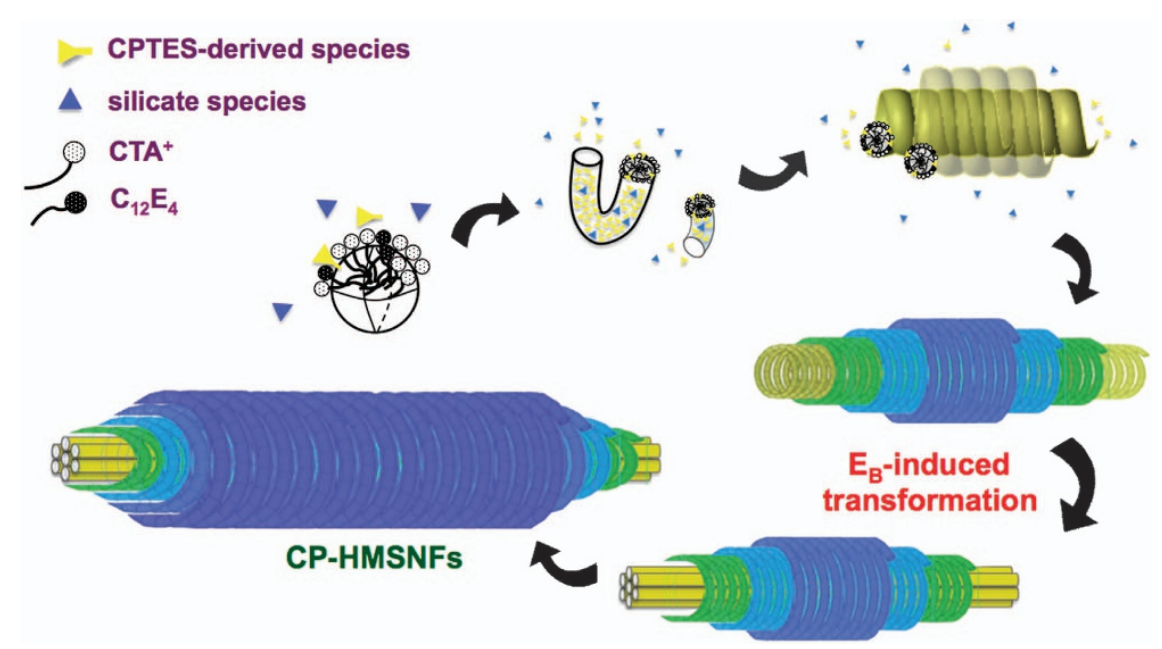

Scheme 1 Scheme 1Schematic representation of the formation of CP-HMSNFs with coaxial helical/columnar mesostructures. CP-HMSNF, chloropropylfunctionalized helical mesoporous silica nanofiber.

total metal loading ( $15 \mathrm{wt} \%)$, particle size, lattice constant and degree of alloying (Supplementary Figure S13A and B). The magnetization-demagnetization curves measured at 5-300 K (Supplementary Figure S13C) indicated nearly identical magnetic properties for the two samples despite the differences in the channel orientation of the host silica (Supplementary Table S1). To the best of our knowledge, such geometry-dependent magnetic transitions have never been observed or studied for geometrically one-dimensional nanomaterials. ${ }^{38}$ Further detailed studies are necessary to elucidate this phenomenon. 

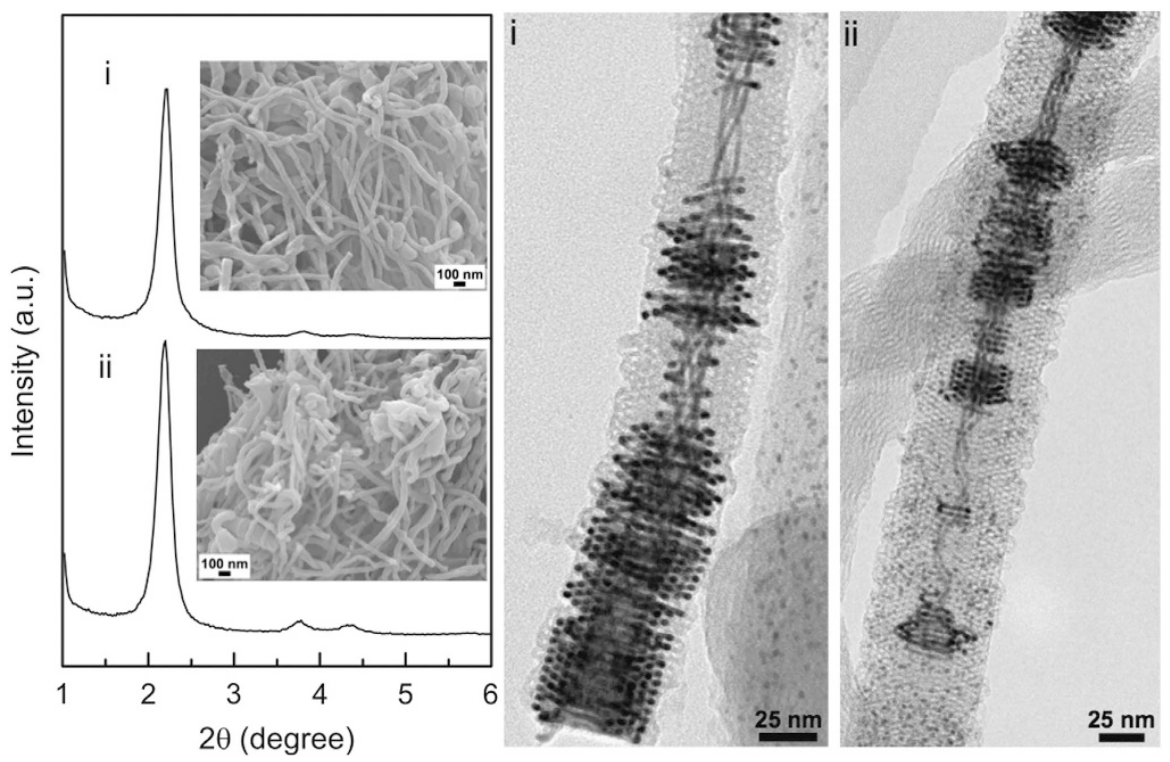

Figure 5 XRD patterns, SEM and TEM images of the samples synthesized with 10\% trifluoropropyltrimethoxysilane (i) or iodopropyltrimethoxysilane (ii) and an injection rate of $3.0 \mathrm{ml} \mathrm{h}^{-1}$. SEM, scanning electron microscope; TEM, transmission electron microscope; XRD, X-ray diffraction.

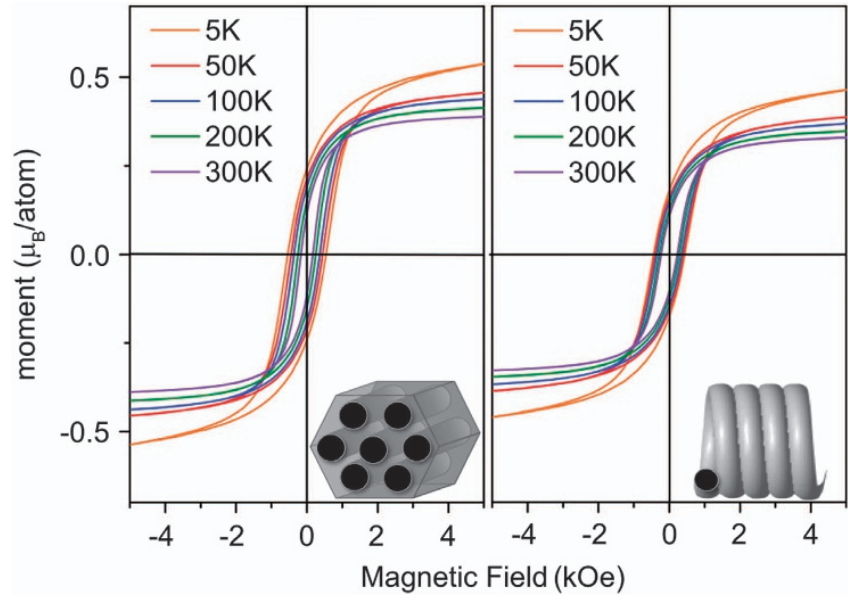

Figure 6 Magnetization-demagnetization curves of $\mathrm{Pt}_{60} \mathrm{C}_{40} @ \mathrm{MCM}-41$ (left) and $\mathrm{Pt}_{60} \mathrm{Co}_{40} @ \mathrm{CP}-\mathrm{HMSNFs}$ (right) measured at $5 \mathrm{~K}, 50 \mathrm{~K}, 100 \mathrm{~K}, 200 \mathrm{~K}$ and $300 \mathrm{~K}$. CP-HMSNF, chloropropyl-functionalized helical mesoporous silica nanofiber.

Table 1 Magnetic properties of $\mathrm{Pt}_{60} \mathrm{Co}_{40} @ \mathrm{CP}-\mathrm{HMSNF}$ and $\mathrm{Pt}_{60} \mathrm{Co}_{40} @ \mathrm{MCM}-41$

\begin{tabular}{|c|c|c|c|c|c|c|c|c|c|}
\hline & \multicolumn{4}{|c|}{$5 K$} & \multicolumn{4}{|c|}{$300 K$} & \multirow[b]{2}{*}{$\Delta H c^{a}$} \\
\hline & Ms & $M r$ & $M r / M s$ & $H c$ & Ms & $M r$ & $M r / M s$ & $H C$ & \\
\hline $\begin{array}{l}\mathrm{Pt}_{60} \mathrm{CO}_{40} @ \mathrm{CP}- \\
\mathrm{HMSNFs}\end{array}$ & 0.469 & 0.180 & $38 \%$ & 442 & 0.338 & 0.110 & $32 \%$ & 225 & $-49.1 \%$ \\
\hline $\mathrm{Pt}_{60} \mathrm{Co}_{40} @ \mathrm{MCM}-41$ & 0.548 & 0.249 & $45 \%$ & 536 & 0.391 & 0.112 & $29 \%$ & 165 & $-69.2 \%$ \\
\hline
\end{tabular}

\section{CONCLUSION}

In summary, we have discovered a confinement-free synthesis of CP-functionalized mesoporous silica nanofibers with coaxial helical/columnar mesostructures. With the organic groups mainly localized at the center, the nanofibers have been applied for the selective inclusion of Pt and/or $\mathrm{Fe}_{2} \mathrm{O}_{3}$. We have also fabricated 3-nmwide ferromagnetic PtCo helical nanowires and displayed the geometry-dependent magnetic properties. The results show the uniqueness of the functional nanofibers and the tremendous opportunities for use in innovative applications.

\section{CONFLICT OF INTEREST}

The authors declare no conflict of interest.

\section{ACKNOWLEDGEMENTS}

We acknowledge Dr L.Z. for his assistance in superconducting quantum interference device (SQUID) measurements. This work is supported by the Ministry of Science and Technology of Taiwan under contract no. NSC1012628-M-007-001-MY2.

1 Snir, Y. \& Kamien, R. D. Entropically driven helix formation. Science 307, 1067-1067 (2005).

2 Yang, S., Zhao, L. Z., Yu, C. Z., Zhou, X. F., Tang, J. W., Yuan, P., Chen, D. Y. \& Zhao, D. Y. On the origin of helical mesostructures. J. Am. Chem. Soc. 128, 10460-10466 (2006)

3 Yang, C. M., Lin, C. Y., Sakamoto, Y., Huang, W. C. \& Chang, L. L. 2D-Rectangular c2mm mesoporous silica nanoparticles with tunable elliptical channels and lattice dimensions. Chem. Commun. (Camb) 45, 5969-5971 (2008).

4 Huang, W.-C., Chang, L.-L., Sakamoto, Y., Lin, C.-Y., Lai, N.-C. \& Yang, C.-M. Kinetically controlled formation of helical mesoporous silica nanostructures correlated to a ribbon intermediate phase. RSC Adv. 1, 229-237 (2011).

5 Che, S., Liu, Z., Ohsuna, T., Sakamoto, K., Terasaki, O. \& Tatsumi, T. Synthesis and characterization of chiral mesoporous silica. Nature 429, 281-284 (2004).

6 Chouaieb, N., Goriely, A. \& Maddocks, J. H. Helices. Proc. Natl Acad. Sci. USA 103 9398-9403 (2006).

7 Huo, Q. S., Zhao, D. Y., Feng, J. L., Weston, K., Buratto, S. K., Stucky, G. D., Schacht, S. \& Schüth, F. Room temperature growth of mesoporous silica fibers: a new highsurface-area optical waveguide. Adv. Mater. 9, 974-978 (1997).

8 Meng, X. J., Yokoi, T., Lu, D. L. \& Tatsumi, T. Synthesis and characterization of chiral periodic mesoporous organosilicas. Angew. Chem. Int. Ed. 46, 7796-7798 (2007).

9 Kamata, K., Suzuki, S., Ohtsuka, M., Nakagawa, M., lyoda, T. \& Yamada, A. Fabrication of left-handed metal microcoil from spiral vessel of vascular plant. Adv. Mater. 23, 5509-5513 (2011). 
10 Wang, Y., Xu, J., Wang, Y. \& Chen, H. Emerging chirality in nanoscience. Chem. Soc. Rev. 42, 2930-2962 (2013).

11 Ren, Z. \& Gao, P.-X. A review of helical nanostructures: growth theories, synthesis strategies and properties. Nanoscale 6, 9366-9400 (2014).

12 Wu, Y., Livneh, T., Zhang, Y. X., Cheng, G., Wang, J., Tang, J., Moskovits, M. \& Stucky, G. D. Templated synthesis of highly ordered mesostructured nanowires and nanowire arrays. Nano Lett. 4, 2337-2342 (2004).

13 Xie, J., Duan, Y. \& Che, S. Chirality of metal nanoparticles in chiral mesoporous silica. Adv. Funct. Mater. 22, 3784-3792 (2012).

14 Yamauchi, Y., Takai, A., Nagaura, T., Inoue, S. \& Kuroda, K. Pt fibers with stacked donut-like mesospace by assembling Pt nanoparticles: guided deposition in physically confined self-assembly of surfactants. J. Am. Chem. Soc. 130, 5426-5427 (2008).

15 Rambaud, F., Vallé, K., Thibaud, S., Julián-López, B. \& Sanchez, C. One-pot synthesis of functional helicoidal hybrid organic-inorganic nanofibers with periodically organized mesoporosity. Adv. Funct. Mater. 19, 2896-2905 (2009).

16 Motohiro, T. \& Taga, Y. Thin film retardation plate by oblique deposition. Appl. Opt. 28, 2466-2482 (1989).

17 Kuzyk, A., Schreiber, R., Fan, Z., Pardatscher, G., Roller, E.-M., Hogele, A., Simmel, F. C., Govorov, A. O. \& Liedl, T. DNA-based self-assembly of chiral plasmonic nanostructures with tailored optical response. Nature 483, 311-314 (2012).

18 Zhang, L., Abbott, J. J., Dong, L., Peyer, K. E., Kratochvil, B. E., Zhang, H., Bergeles, C. \& Nelson, B. J. Characterizing the swimming properties of artificial bacterial flagella. Nano Lett. 9, 3663-3667 (2009).

19 Han, Y., Zhao, L. \& Ying, J. Y. Entropy-driven helical mesostructure formation with achiral cationic surfactant templates. Adv. Mater. 19, 2454-2459 (2007).

20 Yuan, P., Zhao, L. Z., Liu, N. A., Wei, G. F., Wang, Y. H., Auchterlonie, G. J., Drennan J., Lu, G. Q., Zou, J. \& Yu, C. Z. Evolution of helical mesostructures. Chem. Eur. J. 16 1629-1637 (2010)

21 Tottori, S., Zhang, L., Qiu, F., Krawczyk, K. K., Franco-Obregón, A. \& Nelson, B. J. Magnetic helical micromachines: fabrication, controlled swimming, and cargo transport. Adv. Mater. 24, 811-816 (2012).

22 Wang, J., Tsung, C.-K., Hong, W., Wu, Y., Tang, J. \& Stucky, G. D. Synthesis of mesoporous silica nanofibers with controlled pore architectures. Chem. Mater. 16, 5169-5181 (2004).

23 Wu, Y., Cheng, G., Katsov, K., Sides, S. W., Wang, J., Tang, J., Fredrickson, G. H. Moskovits, M. \& Stucky, G. D. Composite mesostructures by nano-confinement. Nat. Mater. 3, 816-822 (2004).

24 Platschek, B., Petkov, N. \& Bein, T. Tuning the structure and orientation of hexagonally ordered mesoporous channels in anodic alumina membrane hosts: a 2D small-angle x-ray scattering study. Angew. Chem. Int. Ed. 45, 1134-1138 (2006).

25 Wang, D., Kou, R., Yang, Z., He, J., Yang, Z. \& Lu, Y. Hierachical mesoporous silica wires by confined assembly. Chem. Commun. 166-167 (2005).

$26 \mathrm{Lu}$, Q., Gao, F., Komarneni, S. \& Mallouk, T. E. Ordered SBA-15 nanorod arrays inside a porous alumina membrane. J. Am. Chem. Soc. 126, 8650-8651 (2004).
27 Platschek, B., Köhn, R., Döblinger, M. \& Bein, T. Formation mechanism of mesostructured silica in confined space: an in situ GISAXS study. Chem. Phys. Chem $\mathbf{9}$ 2059-2067 (2008).

28 Platschek, B., Keilbach, A. \& Bein, T. Mesoporous structures confined in anodic alumina membranes. Adv. Mater. 23, 2395-2412 (2011).

29 Chang, A., Lai, N. -C. \& Yang, C. -M. MCM-48 nanorods: a self-assembled isotropic cubic mesostructure with anisotropic morphology. RSC Adv. 2, 12088-12090 (2012).

30 Lai, N. -C., Lin, C. -Y., Ku, P. -H., Chang, L. -L., Liao, K. -W., Lin, W. -T. \& Yang, C. -M Hollow mesoporous la3d silica nanospheres with single-unit-cell-thick shell: Spontaneous formation and drug delivery application. Nano Res. 7, 1439-1448 (2014).

31 Chen, P. -K., Lai, N. -C., Ho, C. -H., Hu, Y. -W., Lee, J. -F. \& Yang, C. -M. New synthesis of MCM-48 nanospheres and facile replication to mesoporous platinum nanospheres as highly active electrocatalysts for the oxygen reduction reaction. Chem. Mater. 25, 4269-4277 (2013).

32 Li, S. -S., Chang, C. -P., Lin, C.-C., Lin, Y. -Y., Chang, C. -H., Yang, J.-R., Chu, M. -W. \& Chen, C. -W. Interplay of three-dimensional morphologies and photocarrier dynamics of polymer/TiO2 bulk heterojunction solar cells. J. Am. Chem. Soc. 133, 11614-11620 (2011).

33 Brinker, C. J. \& Scherer, G. W. Sol-Gel Science (Academic Press, Inc., San Diego, CA USA, 1990).

34 Entel, P. \& Gruner, M. E. Large-scale ab initio simulations of binary transition metal clusters for storage media materials. J. Phys.: Condens. Matter 21, 064228 (2009).

35 Chen, C. H., Higgins, A. K. \& Strnat, R. M. Effect of geometry on magnetization distortion in closed-circuit magnetic measurements. J. Magn. Magn. Mater. 320, L84-L87 (2008).

36 Merazzo, K. J., Leitao, D. C., Jimenez, E., Araujo, J. P., Camarero, J., Real, R. P. d. Asenjo, A. \& Vazquez, M. Geometry-dependent magnetization reversal mechanism in ordered Py antidot arrays. J. Phys. D: Appl. Phys. 44, 505001 (2011).

37 Kronmuller, H. \& Parkin, S. Handbook of Magnetism and Advanced Magnetic Materials (John Wiley and Sons, Inc., 2007).

38 Chiruta, D., Linares, J., Miyashita, S. \& Boukheddaden, K. Role of open boundary conditions on the hysteretic behaviour of one-dimensional spin crossover nanoparticles. J. Appl. Phys. 115, 194309 (2014).

(i) This work is licensed under a Creative Commons Attribution 4.0 International License. The images or other third party material in this article are included in the article's Creative Commons license, unless indicated otherwise in the credit line; if the material is not included under the Creative Commons license, users will need to obtain permission from the license holder to reproduce the material. To view a copy of this license, visit http:// creativecommons.org/licenses/by/4.0/

Supplementary Information accompanies the paper on the NPG Asia Materials website (http://www.nature.com/am) 